\title{
A fitter code for Deep Virtual Compton Scattering and Generalized Parton Distributions
}

\author{
M. Guidal ${ }^{\mathrm{a}}$ \\ Institut de Physique Nucléaire d'Orsay, 91405 Orsay, France \\ Original article: Eur. Phys. J. A 37, 319 (2008) DOI: 10.1140/epja/i2008-10630-6 \\ Received: 5 March 2009 \\ Published online: 18 March 2009 - (c) Società Italiana di Fisica / Springer-Verlag 2009
}

An unfortunate typographical error (by the author) has slipped into eqs. (7) and (8) of ref. [1]. Although these two particular equations are not essentially used and do not affect any result in the article, we would like, for future references, to correct them.

Equations (7) and (8) of ref. [1] should thus read

$$
\tilde{H}(\xi, \xi, t)+\tilde{H}(-\xi, \xi, t) \quad \text { and } \quad \tilde{E}(\xi, \xi, t)+\tilde{E}(-\xi, \xi, t)
$$

i.e., it should be a sum instead of a difference.

We restate that this was only a typographical error and that the correct formulaes are actually coded in our program.

We are thankful to H. Moutarde who alerted us on this point.

\section{References}

1. M. Guidal, Eur. Phys. J. A 37, 319 (2008).

\footnotetext{
${ }^{a}$ e-mail: guidal@ipno.in2p3.fr
} 